\title{
Karl Polanyi contre Ludwig von Mises
}

Esquisse d'un anti-formalisme « vétéro-autrichien »

Karl Polanyi gegen Ludwig von Mises. Umriss eines "als-österreichischen" AntiFormalismus

Karl Polanyi vs. Ludwig von Mises. Outline of an "Old-Austrian" anti-formalism

\section{Santiago Pinault}

\section{(2) OpenEdition}

\section{Journals}

Édition électronique

URL : https://journals.openedition.org/austriaca/1638

DOI : 10.4000/austriaca.1638

ISSN : 2729-0603

Éditeur

Presses universitaires de Rouen et du Havre

\section{Édition imprimée}

Date de publication : 1 juin 2020

Pagination : 173-195

ISBN : 979-10-240-1492-0

ISSN : 0396-4590

Référence électronique

Santiago Pinault, « Karl Polanyi contre Ludwig von Mises », Austriaca [En ligne], 90 | 2020, mis en ligne le 01 juin 2021, consulté le 30 septembre 2022. URL : http://journals.openedition.org/austriaca/1638 ; DOI : https://doi.org/10.4000/austriaca.1638

Ce document a été généré automatiquement le 30 septembre 2022.

Tous droits réservés 


\title{
Karl Polanyi contre Ludwig von Mises
}

\author{
Esquisse d'un anti-formalisme « vétéro-autrichien » \\ Karl Polanyi gegen Ludwig von Mises. Umriss eines "als-österreichischen" Anti- \\ Formalismus \\ Karl Polanyi vs. Ludwig von Mises. Outline of an "Old-Austrian" anti-formalism
}

\section{Santiago Pinault}

\section{Introduction. Notre "fil rouge »: une " ancienne conversation » entre Polanyi et Mises}

1 Karl Polanyi (1886-1964) a laissé une œuvre inachevée, fourmillante de nombreux textes très courts ou de brouillons, ce qui conduit souvent ses lecteurs à ne s'intéresser qu'à La grande transformation, l'unique livre publié par Polanyi en son nom seul, en 1944 et en anglais (sous le titre The Great Transformation). Ce texte est pourtant assez hétéroclite et son propos véritable reste discuté, comme le reste d'une production intellectuelle qu'il faut plutôt appréhender dans son ensemble, pour le principe même de sa démarche, que dans ses détails.

2 Plusieurs tentatives ont été menées en ce sens, pour comprendre dans sa globalité cette œuvre issue de contextes successifs : commencée en Hongrie (1908-1919), poursuivie en Autriche (1919-1933), puis en Angleterre (d'abord entre 1934 et 1941, puis entre 1944 et 1946), elle se termine en Amérique du Nord (dès 1941 jusqu'à 1943, puis à partir de 1947). Polanyi lui-même se pencha rétrospectivement sur ses propres écrits ${ }^{1}$, tout comme le firent des contemporains ou des proches. Sans doute la meilleure tentative de décryptage fut celle produite par Felix Schafer, son plus proche collaborateur à Vienne entre 1924 et $1933^{2}$.

3 Schafer n'a pas connu la période hongroise de Polanyi. Comme il n'avait pas accès à tous les textes de son ami, il n'a pas pu voir le hiatus entre une première période, de 1908 à 1924, et la suite sa carrière. Schafer avait connu son ami déjà converti au 
christianisme, quoique né dans une famille juive "assimilée ", selon l'appellation de la bourgeoisie juive libérale dans l'empire des Habsbourg ${ }^{3}$.

Or, la religion de Polanyi, peu évidente dans sa teneur, paraît bien primordiale pour comprendre son œuvre: elle nous semble notamment fonder son orientation personnaliste, qui sert à son tour de base à son " socialisme». Schafer a pu intimement lier le christianisme et le socialisme de Polanyi, comme si cela allait de soi. Il s'épargnait ainsi bien des complications. Ces lacunes furent sa chance, car il put analyser quatre décennies de recherches sous l'angle d'une trajectoire cohérente unifiée. Il avait suivi de près les premiers pas de Polanyi depuis Vienne, en NouvelleZélande - où Schafer s'était réfugié, à l'instar d'autres penseurs viennois, comme Karl Popper. Schafer put montrer l'esprit viennois cristallisé dans l'œuvre de Polanyi sans partager certains biais idéologiques des proches de Polanyi. Ainsi, pour peu que l'on se convainque qu'il y eut effectivement un basculement irrémédiable dans la pensée de Polanyi entre 1922 et 1924, Schafer se trouverait offrir au final l'unique grille de lecture satisfaisante. Cela n'exclut pas que la continuité soit problématique dans l'œuvre polanyienne, et dès lors une exégèse plus avancée de l'œuvre de Polanyi est requise, audelà de celle encore balbutiante à divers égards plus de cinquante ans après sa mort. La question complexe que nous posons dans les pages qui suivent, à savoir celle du rapport de Polanyi à Ludwig von Mises dans la perspective de l'héritage de l'école autrichienne d'économie, pourrait au moins offrir une interprétation alternative crédible.

L'année 1922 correspond pour Polanyi au début d'un long débat théorique avec Mises. C'est en réaction à celui-ci que Polanyi tient, en 1924, un séminaire presque entièrement dédié à répondre à l'« Autrichien ». Aux côtés de Polanyi, Schafer saisit l'importance philosophique des enjeux de leurs discussions, plus profonds même que les divergences politiques auxquelles les commentateurs s'arrêtent trop souvent. L'opposition à Mises, vu comme un penseur paradigmatique de la rationalité formelle, pas simplement comme un "libéral», devait selon nous constituer le fil rouge des recherches de Polanyi. Nous trouvons les preuves de cette intuition dans l'exploration des archives, en nous inspirant de la perspective tracée par Schafer, tout en nous en émancipant.

Dans une première partie, nous présentons comment, dans la «période viennoise » de la carrière de Polanyi, le débat entamé avec Mises structure ses recherches. Une deuxième partie refuse la progression linéaire qui structurait la vue de Schafer sur son ami, et montre que c'est pour abandonner l'approche historique suivie dans The Great Transformation que Polanyi décida, entre 1946 et 1960, de réinscrire le débat originel avec Mises dans des catégories qu'il avait trouvées par ailleurs chez Max Weber. La troisième partie montre comment, dans sa "période nord-américaine", Polanyi a esquissé (seulement) un paradigme que nous nommerons «mengero-wébérien » (en référence au fondateur de l'école autrichienne, Carl Menger, comme à Weber, déjà cité) : Polanyi peut y intégrer le formalisme de Mises et opposer en retour à ce dernier deux figures importantes, en s'appuyant sur des bases solides, pour continuer leur débat entamé à Vienne un demi-siècle auparavant. 


\section{Polanyi en Autriche (1922-1932) : formalisme misésien ou humanisme chrétien}

7 Polanyi part de Hongrie pour arriver à Vienne en juin 1919, apparemment pour des raisons de santé ${ }^{4}$, après avoir travaillé quelques mois pour le Commissariat populaire de la production sociale du régime bolchévique de Béla Kun. Mises est alors, quant à lui, consultant pour le gouvernement autrichien ${ }^{5}$, quand les deux hommes se croisent pour la première fois :

Mises mena des négociations avec des représentants du neuf ([mais] bref) gouvernement communiste en Hongrie, concernant les droits de propriété des citoyens autrichiens en Hongrie. Le chef de la délégation hongroise [...] prit très rarement part aux réunions et les réels débatteurs du côté hongrois furent plutôt un certain Dr. Görög et un certain Dr. Polanyi. [...] Ce Polanyi, qui avait un esprit brillant et qui était convaincu par le communisme, se heurta de nombreuses fois dans les réunions avec Mises, souvent dans de longues discussions concernant des questions fondamentales en philosophie sociale ${ }^{6}$.

La fougue de Polanyi ressemble passablement au zèle d'un nouveau converti puisque, de novembre 1918 à mars 1919, lui et d'autres membres de sa famille étaient associés de près au régime républicain du comte Károlyi, dans la ligne du parti « civique-radical » dont Polanyi avait été un leader dès sa création en 1914. Encore en décembre 1918, Polanyi publiait un article anti-socialiste, et il demandait, en janvier 1919, à la «classe manuelle» de suivre la "classe intellectuelle» en affichant clairement son antibolchevisme. Le ralliement de Polanyi au communisme était donc tout récent au moment indiqué par la citation, ce qui explique peut-être de la virulence forcée, qui contribua à créer en retour les conditions d'un débat au tour trop personnel avec Mises.

À partir de 1920, Polanyi demeure dans un exil définitif en Autriche. Il se met à écrire un livre dont les différents brouillons qui nous restent montrent qu'il devait traiter notamment de la scientificité en politique. Mises publie, quant à lui, l'article qui ouvre le débat devenu fameux à propos du " calcul économique en économie socialiste ». Il y pose le principe qu'une société complexe ne peut en aucun cas se passer d'un système de prix pour fixer la valeur des biens à échanger. Pour être significatifs, ces prix doivent émaner de la confrontation des offres et des demandes sur un marché libre, la monnaie étant indispensable aux échanges de marchandises. Pour Mises, prix, marché et monnaie sont trois institutions aussi indissociables que nécessaires. Dès lors, toute une génération de penseurs opposés au marché ou au "règne de l'argent » se met à tenter de répondre à l'affirmation de ce principe. Dans ce cadre, en mai 1922, Polanyi publie les 43 pages de son article "Sozialistische Rechnungslegung" dans la prestigieuse revue Archiv für Sozialwissenschaft und Sozialpolitik (re)fondée entre autres par Max Weber.

Dans son texte, Polanyi expose les grands traits d'un modèle théorique de comptabilité au sein d'une "Gemeinschaft " économique où la préférence va à des processus démocratiques plutôt qu'à la coordination «aveugle » des ressources par les échanges sur les marchés. Ce choix « démocratique » donne du poids aux décisions que les êtres humains prennent pour eux-mêmes en toute conscience dans les échanges qui les concernent, sans pour autant sacrifier leur liberté en tant que personnes composant la société. Polanyi reste dans un cadre de mesure quantifiée des coûts et des profits; il s'oppose à ceux qui rejettent les outils de quantification. Ceux-ci étaient bien entendu déclarés indispensables par Mises, mais Polanyi tient à remplir un cadre présenté par ce 
dernier, qu'il juge froid et abstrait. Invoquant des qualités irréductibles aux seuls chiffres, les qualités "touchant à l'humain", il vise à humaniser ce cadre par une mise sous tutelle de l'économique par le politique. Nous sommes au cœur du débat: Mises entend réduire à leur plus petit dénominateur commun les variables et entités abstraites de son modèle pour qu'elles soient universelles et, à ce prix, il tente de trouver les outils neutres et dépolitisés permettant de gérer les biens à échanger dans la communauté. Il tente de faire l'économie des questions touchant aux rapports de domination, au pouvoir, débats sans fin dans les négociations humaines ou facteurs d'une violence souvent effective. De son côté, pour des raisons morales (religieuses) et à l'aune de son exigence de réalisme anthropologique, Polanyi ne peut guère accepter ni qu'il faille que les êtres humains soient atomisés et " objectivisés ${ }^{7}$ » artificiellement, ni que les personnes soient comme dépossédées d'elles-mêmes au sein d'institutions qu'elles produisent, mais qui leur échappent.

Gratifié d'une réponse personnelle par Mises dans un article publié dans l'Archiv für Sozialwissenschaft und Sozialpolitik en 1923, Polanyi lui écrit une lettre privée en février $1924^{8}$ et lui répond publiquement dans deux articles, "Die funktionelle Theorie der Gesellschaft und das Problem der sozialistischen Rechnungslegung (1924), puis « Neue Erwägungen zu unserer Theorie und Praxis » (1925). Parallèlement, Polanyi tient un séminaire chez lui, entre 1924 et 1932, auquel participent notamment Schafer et Popper. Le but principal de ce séminaire est de poursuivre la discussion du modèle de 1922, afin de concevoir une Gemeinschaft capable d'exercer un autocontrôle d'ensemble, sans verser dans une organisation totalitaire pour autant, tout en prenant en compte les objections émises par Mises en 1922 quand celui-ci doutait que le modèle de Polanyi pût constituer une « troisième voie » entre l'économie de marché et le communisme.

En rejetant le marxisme scientifique ${ }^{9}$, Polanyi et son groupe situent leurs réflexions sur le terrain autrichien de Mises. Le groupe de réflexion travaille sur le Zurechnungsproblem en lisant Carl Menger, ainsi que Eugen von Böhm-Bawerk, Friedrich von Wieser et Hans Mayer, tous trois considérés comme héritiers intellectuels de plein droit de Menger au même titre que Mises. La quête d'une «troisième voie " situe le débat entre les notions de Kaufkraftwirtschaft wieserienne et de Tauschwirtschaft de Böhm-Bawerk (que nous n'explorerons pas ici). En 1928, Schafer et Polanyi rédigent 28 pages d'un texte où ils critiquent la solution de Hans Mayer, mais sans l'achever. Six ans plus tard, lorsque Polanyi émigra en Angleterre pour fuir le régime d'Engelbert Dollfuss, il était devenu rédacteur en chef du prestigieux journal Der Österreichische Volkswirt, mais il n'avait toujours pas publié la deuxième version de son modèle de 1922, ou répondu sur le fond aux critiques alors émises par Mises.

\section{Polanyi entre la Grande-Bretagne et l'Amérique du Nord (1934-1945) : sociologie chrétienne et anti- progressisme}

13 À partir du milieu des années 1930 et depuis le monde anglo-saxon où il évolue désormais, Polanyi travaille à une sociologie que l'on peut qualifier de chrétienne, à l'aune du même personnalisme que celui forgé à Vienne, mais en se situant sur un plan plus philosophique. Il s'agit, pour lui et ses camarades chrétiens socialistes ou communistes, de penser une sociologie égalitariste, cette fois sur fond d'essor des idéologies holistes fondant les totalitarismes qui submergent le monde de langue 
allemande. Polanyi et ses proches visent une doctrine qui ne soit ni un individualisme atomiste - contre les « libéraux de l'école de Mises » donc ${ }^{10}$ - ni un holisme collectiviste - contre les auteurs historicistes ou encore les vitalistes, comme Ludwig Klages ou Othmar Spann. Le but affirmé est de relier l'esprit du christianisme et le socialisme, dans une inspiration qui peut rappeler celle du jeune Marx, notamment dans les textes de 1844 .

En 1944, dans The Great Transformation, Polanyi recueille les fruits de la réflexion menée jusque-là en suivant trois approches différentes, avec pour effet de brouiller son propos.

Dans la première, de nature théorique et universaliste, Polanyi dresse (principalement dans le chapitre 3) une antinomie entre le progrès de la Raison - présenté par le terme d'«Amélioration", qui permet notamment en pratique l'industrialisation - et les environnements social et naturel de l'être humain (ce qu'il appelle l'« Habitation»). Selon Polanyi, l'industrialisation a suscité de fait un monde de nature au fond "satanique ", où l'Homme perd toujours plus pied, victime de sa chute originelle. Nous utilisons à escient les majuscules pour un tel discours, où Polanyi prend des accents messianiques: le lecteur peut penser que l'image sous-jacente est celle du fruit de l'Arbre de la connaissance dont la consommation aurait condamné pour ainsi dire l'humanité à sceller à chaque étape de son développement un pacte faustien qui la lie toujours plus au monde de la Machine.

Dans la deuxième approche, historique et centrée sur l'histoire, relue du xVIII ${ }^{e}$ au $\mathrm{xx}^{\mathrm{e}}$ siècle dans un contexte anglo-saxon, Polanyi s'en prend au modèle social de «la société de marché » et à l'idéologie dite « libérale », qui la sous-tend. Polanyi prend le contrepied exact des positions de Mises, notamment en attaquant notamment l'étalonor que Mises défendait, et en inversant le plus souvent purement et simplement les arguments misésiens, même lorsqu'il englobe le penseur autrichien dans un ensemble bien plus large de penseurs « libéraux ${ }^{11} »$ :

Des auteurs libéraux comme Spencer et Sumner, Mises et Lippmann, proposent une vision [...] substantiellement similaire à la nôtre, mais ils l'interprètent de manière complètement différente. Alors que, pour nous, le concept de marché autorégulateur est utopique et que son progrès a été stoppé par l'autoprotection réaliste de la société, pour eux le protectionnisme était une erreur due à l'impatience, à la cupidité et à l'absence de vision à long terme, terme qui constitue l'échelle de temps à laquelle le marché aurait résolu tous leurs problèmes ${ }^{12}$.

17 La dualité de ces approches est reflétée dans les différents titres que Polanyi a voulu donner à son projet. Dans l'un, il parlait des «origines du cataclysme ${ }^{13}$ ", et les dernières pages de son livre montrent que Polanyi inscrit la temporalité de cette "déroute » des cent cinquante années précédentes dans le temps long de trois grandes phases de l'Histoire, selon un schéma historiciste allusif, peut-être (pseudo-)chrétien. Un autre titre évoquait l'« utopie libérale ${ }^{14}$ ».

18 L'approche précédente paraît cependant plus fine et intéressante. L'ouvrage se clôt précisément sur la question de "la liberté dans une société complexe " (The Great Transformation, chap.21). C'est la question que Polanyi a voulu reprendre ensuite, notamment dans le texte dont il a entamé la rédaction entre 1956 et 1959 : Freedom and Technology.

19 Enfin, la troisième approche présente dans The Great Transformation, texte aussi riche d'intuitions qu'ambigu, est explicitement comparatiste. Polanyi oppose le modèle 
occidental à des Gemeinschaften non occidentales et non industrialisées, en se situant dans le sillage des anthropologues Richard Thurnwald, Bronisław Malinowski et Melville Herskovits. Cette approche confronte une sorte de "paradis perdu " à l'évolution de l'Occident depuis le XvIII ${ }^{\mathrm{e}}$ siècle, mais pour exotique et séduisante qu'elle ait pu paraître à maints lecteurs, cette approche s'intègre mal à la perspective des deux premières.

20 En effet, si l'idée d'une «Chute » de l'humanité, due aux douleurs de l'enfantement de la modernité technologique (à savoir, le fruit le plus avancé de la connaissance) a fait entrer l'homme occidental inexorablement dans l'Histoire, nul retour en arrière n'est possible. Il faut soit poursuivre dans la fuite en avant, dans l'attente du temps messianique où celle-ci serait abolie (une " fin des temps » réinstaurant l'état de félicité initial), soit résoudre dialectiquement l'antinomie entre le Progrès et l'« Habitation " humains. En aucun cas, les exemples pris tout au long du volume à l'extérieur de l'Occident - qui ne sont donc pas concernés par l'industrialisation, par la croissance du capital et des grandes métropoles, par la division du travail rendue nécessaire par la complexité des connaissances et techniques - ne peuvent donner de clefs pour comprendre un monde dans lequel ces réalités existent dorénavant ${ }^{15}$.

21 Ne tient guère plus l'idée alors en vogue que les dirigeants occidentaux auraient créé, depuis la fin du XvIII ${ }^{e}$ siècle, une société «malade » de son utopie modernisatrice, non viable à long terme, et appelée à revenir à la "normale " après la seconde guerre mondiale, cette dernière n'ayant été que le dernier symptôme de sa pathologie. La solution au problème politique créé par l'industrialisation et le choix des élites libérales en faveur d'une "société de marché " peut se résoudre soit par un geste pleinement réactionnaire, ce à quoi Polanyi se refuse quoiqu'il doute de l'aspect bénéfique du "Progrès ", soit par la recherche, encore, d'une dialectique où l'« ancien régime » politique prémoderne intégrerait d'inéluctables contraintes nouvelles inhérentes à la (post-)modernité. Dans un cas comme dans l'autre, les idées occidentales - notamment pour expliquer la réaction spontanée des peuples face à l'utopie de la "société de marché » - permettent à elles seules de penser une entreprise d'aggiornamento de l'héritage des Lumières ou bien une réaction d'inspiration romantique "antimoderne ", et ce, sans recourir à ce que Polanyi considère comme de fausses solutions de nature collectiviste, fasciste ou soviétique.

Néanmoins, bien que superflus dans l'argumentation de The Great Transformation, les exemples empiriques donnaient du contenu au problème théologico-philosophique de l'antinomie mise à jour dans la première approche, et réinscrivait ses enjeux non plus dans une perspective historique remontant à l'Angleterre d'Adam Smith et David Ricardo ${ }^{16}$, mais dans une perspective universelle et anhistorique. Polanyi choisit donc d'élargir son champ d'investigation et c'est dans cette optique que Weber devient central dans ses considérations, Polanyi trouvant chez ce dernier les catégories qui lui permettent dorénavant de structurer sa pensée, alors qu'il ne lui accordait auparavant qu'une place marginale dans ses références. Alors qu'il rejoint l'université de Columbia au titre de professeur d'économie, il se met à relire tout Max Weber ${ }^{17}$.

Cet aboutissement qui fait que Polanyi retrouve l'œuvre de Weber à cette étape de sa propre évolution, à la fin de 1946 et au début de 1947, ne constitue pas une avancée pour Polanyi, cependant, sinon un retour à des lectures viennoises d'avant 1922, moment où il lisait patiemment de nombreux sociologues, et notamment, outre Weber, Georg Simmel, Alfred Vierdankt, Ferdinand Tönnies, Franz Oppenheimer ou Leopold 
von Wiese. C'est pourtant à l'occasion de ce retour aux sources, en découvrant si tard sans doute Wirtschaft und Gesellschaft ${ }^{18}$, qu'il trouve enfin des instruments adéquats nous soutenons que cette lecture constitua alors véritablement son «chemin de Damas ».

\section{L'esquisse d'un paradigme vétéro-autrichien produit en Amérique du Nord (1946-1960)}

24 L'influence de Weber devient capitale. Si Polanyi reconnaissait, dans The Great Transformation, à celui-ci d'« avoir été le premier [...], à protester contre ceux qui pensaient que les économies primitives n'aidaient pas à comprendre les motifs et les mécanismes de la société moderne ${ }^{19}$ ", il ne le citait plus ensuite dans son texte. Une fois à Columbia, il trouve chez lui l'ensemble complet et universel de concepts sociologiques fondamentaux dont il était en quête, de la notion d'idéal-type à de nombreux autres concepts qui allaient lui permettre de reprendre son débat avec Mises.

Toutefois, comme en témoignent les archives, plutôt que de mener une lente analyse cursive approfondie des travaux de Weber, Polanyi souhaite plutôt faire œuvre originale quasiment immédiatement, et poursuivre son « ancienne conversation » avec Mises $^{20}$. De fait, les archives prouvent que Polanyi avait lu au moins trois des quatre ouvrages publiés par Mises en allemand ${ }^{21}$. Polanyi s'installe sur un terrain très «autrichien»: il intègre même à ses propres concepts une notion d'inspiration misésienne évidente, la «triade catallactique ». Nous y reviendrons. Surtout, comme à Vienne, Polanyi est de plain-pied avec les conceptions issues de Menger et Wieser, qu'il oppose à Mises et à Böhm-Bawerk, selon une intuition que les historiens de la pensée économique tendent à valider ${ }^{22}$.

\section{Une inspiration tirée de Weber comme de Menger}

Lors de ses travaux à Columbia, Weber figure au premier plan des préoccupations de Polanyi, plus encore que Menger même, en dépit du fait qu'il affiche volontiers sa filiation avec ce dernier pour des raisons stratégiques et polémiques. Après le "montage historique » que fut The Great Transformation en 1944, Polanyi emprunte des concepts à ses prédécesseurs et part en quête de preuves empiriques montrant, idéalement, qu'une société peut évoluer sans marché ou, à défaut, sans que le marché soit l'institution centrale du groupe social. Il tente de montrer que d'autres formes concurrentes ou complémentaires de l'échange monétaire sont également possibles. Ce qu'il n'avait pas apporté sur le plan théorique entre 1922 et 1932, ce qui ne fonctionnait sur le plan historique qu'au prix d'une sélection discutable (selon nous) de faits en 1944, Polanyi le cherche dès lors en multipliant les exemples de modèles de société, sans en trouver vraiment qui lui conviennent tout à fait. La raison de cet échec relatif et de cette quête incessante était que, si ses exemples empiriques présentaient bien des modes d'allocations des ressources sans échange monétaire ou sans marché, le régime politique leur correspondant ne permettait pas d'établir grâce à eux un modèle respectant les postulats éthiques chrétiens, universalistes, égalitaristes et démocratiques qui étaient ceux de Polanyi ${ }^{23}$. 
Polanyi reprend surtout à Weber la distinction entre deux types de rationalité, la rationalité «formelle» et la rationalité "matérielle». Cependant, là où Weber l'applique autant à l'économie qu'à la sociologie et au droit ${ }^{24}$ (mais aussi à la religion, voire à l'art avec la musique), Polanyi reprend cette idée exclusivement au titre de l'économie ${ }^{25}$. Chez Weber, le type formel de rationalité appliquée à l'économie semble assez simple à définir. Weber écrit :

Formal "rational" soll ein Wirtschaften je nach dem maß heißen, in welchem die jeder wie rationalen Wirtschaft wesentliche "Vorsorge" sich in zahlenmäßigen, "rechenhaften", Überlegungen ausdrücken kann und ausdrückt (zunächst ganz unabhängig davon, diese Rechnungen technisch aussehen, ob sie also als Geld- oder als Naturalschätzungen vollzogen werden.) Dieser Begriff ist also (wenn auch, wie sich zeigen wird, nur relativ) eindeutig wenigsten in dem Sinn, daß die Geldform das Maximum dieser formalen Rechenhaftigkeit darstellt (natürlich auch dies: ceteris paribus!)

Le second type de rationalité, de nature « matérielle», est en revanche équivoque :

Dagegen is der Begriff der materialen Rationalität durchaus vieldeutig. Er besagt lediglich dies Gemeinsame: daß eben die Betrachtung sich mit der rein formalen (relativ) eindeutig feststellbaren Tatsache: daß zweckrational, mit technisch tunlichst adäquaten Mitteln, gerechnet wird, nicht begnügt, sondern ethische, politische, utilitaristische, hedonistische, ständische, egalitäre oder irgendwelche anderen Forderungen stellt und daran die Ergebnisse des - sei es auch formal noch so „rationalen“, d.h. rechenhaften - Wirtchaftens wertrational oder material zweckrational bemißt ${ }^{26}$.

Cette distinction de deux orientations (Richtungen) de la rationalité chez Weber est parallèle à celle des «Typen» de l'économie chez Menger ${ }^{27}$. L'esprit de ces travaux est proche de ce que Polanyi reprend avec les paires de notions « économistique/sociétal » "formel/institutionnel», ou encore "formel/substantif», "économie formelle/ économie empirique ». On trouve ces termes dans l'article " The Economy as Instituted Process » de 1957 ou dans ses notes ${ }^{28}$.

Weber pensait que la rationalité (économique) n'est pas univoque, qu'elle concerne autant des techniques de mises en adéquation pratique des moyens avec des fins que des besoins et des choix humains, ce qui est traitable par les mathématiques et un élément qui échappe à tout réductionnisme quantificateur. Ce faisant Polanyi retrouve, au fond, une épistémè du XIX siècle, alors que l'économie n'avait pas encore émergé comme domaine séparé du politique et de la sociologie naissante, avant donc sa mathématisation.

31 En prenant une position "matérielle » de l'approche "sociétale » ou institutionnelle, face au formalisme " économistique » (ou «catallactique») de Mises (et qu'il attribue aussi à Weber), Polanyi se sent la vocation de développer un courant «matériel»/«substantiviste». Comme la définition du formalisme chez Weber correspond à la position de Mises, la façon qu'a Polanyi de concevoir le formalisme paraît identique. L'opposition est très claire si on lit les passages que Weber accorde à la «rationalité matérielle» dans Wirtschaft und Gesellschaft dans cette intention ${ }^{29}$. En revanche, et comme le font à raison tous les traducteurs ${ }^{30}$, Polanyi nomme le second type de rationalité « substantif» (plutôt que « matériel»), afin d'en établir la théorie en ajoutant (selon nous, car il n'est pas explicite ici) des éléments où l'aspiration à une morale chrétienne est sensible.

32 À Menger, Polanyi reprend la distinction des "deux sens du terme "économie" ", qui apparaît en 1948 dans ses cours (archive 31/04, p. 9-12). Pour des raisons stratégiques visant la polémique avec Mises, Polanyi souligne à foison son emprunt au père de 
l'école autrichienne, cette distinction en plaçant sa distinction "formel/substantif " dans le sillage de ce qu'il relève des " deux sens du terme "économie" » du chapitre IV de l'édition posthume de 1923 des Grundsätze de Menger ${ }^{31}$. Polanyi a lu le travail édité par le fils de Menger, et s'il modifie encore le nom des catégories, il reproduit leurs caractéristiques. Chez Polanyi, cela donne le couple «sens formel»/ «sens substantif ", là où Menger parlait de " technisch-ökonomische Disposition » et de "sparende Richtung » (ou « ökonomisierende Richtung ») 32 .

En distinguant ces sens, Menger désignait, d'une part, le point de vue pratique de l'économie, qui concerne l'adéquation de moyens bornés à un environnement donné (de ressources et d'information limitées, par exemple) aux fins définies par l'agent économique, et d'autre part, un point de vue s'attachant à considérer le sens de chaque acte économique (ou de chaque bien économique ou service), indépendamment de la question de la rareté ou des difficultés pratiques de son obtention.

Pourtant, pas une seule fois Polanyi ne cite les définitions wébériennes ou mengeriennes dans son texte. Ses collègues américains ne pouvaient vérifier ses dires sur Menger qu'à condition de lire l'original en allemand ${ }^{33}$. Polanyi adapta ces notions à son propre cadre conceptuel, ce qui n'est pas illégitime puisqu'il juxtapose les distinctions wébérienne et mengérienne, mais qui lui permet de lire la seconde branche de la distinction à sa guise (au risque de commettre un contresens quant au texte d'origine). Polanyi forge de la sorte l'outil dont il avait besoin : dès 1947, il fait de Mises un porte-drapeau du «formalisme» via un concept de "triade catallactique » (commerce, monnaie et marché) que Polanyi lui impute ${ }^{34}$.

Cela se passe quelques mois à peine avant la publication de Human Action par Mises, qui consacre une partie entière à la « catallactique, ou science économique de la société de marché ${ }^{35}$ ». La triade polanyienne correspond presque aux trois institutions que Mises liait en 1920, si ce n'est que le " commerce » a remplacé les "prix ». Un des titres que Polanyi envisageait à l'époque pour le projet que nous connaissons depuis 1977 sous le nom The Livelihood of Man était Redistribution and exchange, Trade, money and markets in Antiquity, où on peut reconnaître dans «redistribution» l'équivalent de la "Kaufkraftwirtschaft» de Wieser et dans "exchange» le "mode d'intégration» privilégié de la "Tauchwirtschaft» de Böhm-Bawerk. Comme Schafer l'avait compris, Polanyi use de nouvelles appellations dans les années 1950, mais il garde quasiment la façon de penser du temps où il affrontait Mises.

36 Cependant, Polanyi a évolué sur la question de la légitimité même du calcul économique, puisqu'en remplaçant les prix par le commerce, il supprime de fait la réflexion sur le prix proprement dit. Ce n'est pas un détail, lorsqu'on se souvient que dans son article sur la "comptabilité", Polanyi cherchait à calculer un prix qui fût décidé de manière politique, et non qui aurait résulté d'un processus autonome de marché. Cela montre qu'il ne s'agit plus pour lui de mettre la calculabilité au service de l'homme, mais de la critiquer pour elle-même, avec la notion d'économie, et de trouver des moyens non quantifiés de gérer les ressources nécessaires à la vie humaine. Le projet qui consistait à mettre des outils formels au service d'un modèle "substantif " (dans l'esprit, même s'il n'employait pas encore ce terme), et ce en quoi résidait le défi de sa troisième voie, semble donc abandonné. Comme l'avait prédit Mises.

37 Dans les années 1950, Polanyi n'entendait donc plus faire aucune concession au formalisme. Toutefois, puisqu'il n'accompagne toujours pas sa réflexion d'un modèle politique qui rendrait superflue l'action du marché «faiseur de prix », il n'a en réalité 
pas avancé d'un iota dans sa réflexion sur cet aspect. Cela peut s'expliquer par son refus de tout modèle autoritaire - du type de ceux qu'il trouve dans les groupes sociaux dits «primitifs » qu'il étudie empiriquement, alors même qu'il reste aveugle à leur violence constitutive (ou qu'il feint de ne pas la voir) ${ }^{36}$. Polanyi n'a pas développé de solution pratique qui inscrirait sa réflexion soit, par exemple, dans une "éthique de l'amour " anarchisante inspirée des Évangiles (éloge des qualités non quantifiables, amour ou effacement des dettes par le pardon), soit dans les modèles d'une Gemeinschaft industrielle (du type socialiste utopiste à la Robert Owen).

De son côté, Mises poursuit en 1949, avec Human Action ${ }^{37}$, l'approche subjectiviste de Menger, pour aboutir à une science plus seulement économique, mais à la "praxéologie ", la "science des actions effectuées par les agents » formels, une science formalisée au moyen de sa méthode dite "a-prioristique ", secondée par la science des échanges appelée "catallactique». Si la réflexion de Polanyi semble stagner, voire reculer sur la question de la calculabilité des actions sociales, celle de Mises progresse à grands pas. Et ce alors que leurs positions sociales respectives se sont inversées entre le rôle qu'ils jouent désormais à New York au regard de celui tenu à Vienne jadis: le professeur d'université est maintenant Polanyi et Mises tient avec peine un séminaire négligé.

Néanmoins, Polanyi, en transportant outre-Atlantique les concepts hérités de deux textes posthumes d'auteurs germanophones, a trouvé un sol plus fécond. Les Grundbegriffe de la sociologie wébérienne lui permettent même d'esquisser un paradigme où le (sous-)paradigme misésien, la branche "formaliste ", est opposé à la branche "substantiviste", dont lui et ses collègues new-yorkais avec qui il publie en 1957 Trade and Markets ${ }^{38}$ seraient les meilleurs représentants. Associées à celles de Weber, les vues de Menger portent les caractérisations de ces deux branches: « formaliste " / « catallactique » et « substantiviste ». Lorsque l'on rappelle la proximité de Weber et de l'école autrichienne, en particulier avant que Menger se retire de la vie publique en 1903 pour continuer de travailler à rééditer ses Grundsätze, le projet de Polanyi, visant à lier Weber et Menger, semble légitime et perspicace.

\section{Un paradigme « vétéro-autrichien »}

Notons que Polanyi n'utilise jamais l'expression d'« école autrichienne d'économie ». $\mathrm{Au}$ regard de ce que celle-ci allait devenir à travers ses autoproclamations successives ${ }^{39}$, un retour à l'esprit viennois d'où vient cette tradition peut sembler pertinent. En particulier dès lors que l'extension des vues autrichiennes va non seulement à Weber, mais plus largement à la sociologie de langue allemande d'une époque où l'économie n'était artificiellement séparée ni de la politique, ni de la société, ni d'une réflexion sur le fait religieux. À ce titre, le projet polanyien était peut-être même nécessaire à revivifier la tradition autrichienne. Elle serait l'esquisse d'un paradigme «mengerowébérien ».

L'origine de l'école autrichienne, mâtinée de sociologie, s'opposerait frontalement aux développements américains de la branche misésienne de l'école autrichienne, notamment à celui de Murray Rothbard, à savoir l'« apriorisme» extrême. Dans les années 1950, Polanyi retourne à la source d'une sociologie intégrant des concepts misésiens dans ce paradigme mêlant Weber et Menger, avant que Rothbard radicalise, dès les années 1960, la pensée de Mises dans un contexte tout différent. 

différentes de celles issues de la Mitteleuropa. Gilles Campagnolo le nomme même " austro-américain ${ }^{40}$ ", pour indiquer qu'il appartient à une école à part entière, si différente qu'elle doit être séparée de l'école née en Autriche avec Menger. Face à ce qui peut apparaître comme une fuite en avant "néo-autrichienne " (un radicalisme partisan exacerbé), l'intuition de donner une suite à l'école autrichienne des origines, en effectuant un retour en arrière et en donnant un élargissement nécessaire à cette tradition, ce dont Polanyi avait eu l'intuition de son côté, constitue peut-être le plus grand apport de ce dernier. Nous proposons d'appeler "vétéro-autrichien" ce paradigme ébauché qui mêle Weber et Menger de manière indissociable, pour bien signaler le retour aux premiers temps de la tradition ${ }^{41}$. l'emporter dans son " ancienne conversation » avec Mises, en retournant contre celuici la légitimité du père de l'école qui lui adressait ses louanges, plutôt que de reprendre les éléments fournis par les membres de cette école, Polanyi reprend seulement, un tiers de siècle après leur parution, les Grundsätze der Volkswirtschaftslehre dans l'édition de 1923 de Menger et Wirtschaft und Gesellschaft de Weber (textes réunis en 1921). Or selon nous, la comparaison de ces deux textes à The Livelihood of Man fait voir un appauvrissement des notions que ne compensent ni la multiplicité d'exemples empiriques apportés à l'appui de la réflexion ni la distinction entre les formes d'«intégration socio-économique» (la catégorisation échange, réciprocité et redistribution $)^{42}$. Ainsi, au lieu de citer ses sources pour s'en inspirer plus ouvertement et produire un travail cumulatif, Polanyi reprend à son compte la tâche de donner une définition unique du sens "substantif» de l'économie pour une des branches de l'économie politique; or il y reste une grande imprécision :

Le sens substantif souligne ce fait élémentaire que les êtres humains, comme tous les autres êtres, ne peuvent exister, à aucune échelle de temps, sans un environnement physique qui les nourrit ; c'est l'origine de la définition substantive de l'économique ${ }^{43}$.

Dans un article publié en 1957, Polanyi reprend ainsi sa formulation :

Le sens substantif de l'économie provient de la dépendance de l'être humain à la nature ainsi qu'aux autres êtres humains. Il fait référence aux échanges mutuels entre l'être humain et son environnement naturel et social, en tant qu'ils ont pour résultat de lui fournir les moyens de satisfaire ses désirs matériels ${ }^{44}$.

Par la suite, dans ce qui allait devenir The Livelihood of Man, les pages où Polanyi développe rapidement les notions d'interactions ou de "mouvements» (changement de lieu de production, de distribution et d'échange, ou changement de propriétaire) des biens, apportent-elles plus à cette définition? Il est permis d'en douter, dès lors que seules «les formes d'intégration » sont déterminées de façon concrète, mais qu'elles laissent pourtant place à plus de questions qu'elles n'en résolvent. La solution toujours cherchée par Polanyi qui pourrait garantir la liberté individuelle au sein d'une "Gesellschaft » complexe et ouverte, même fragmentée en nombreuses «Gemeinschaften » industrielles, n'est pas formulée.

46

Dans le paradigme que Polanyi esquisse, ce qu'il défend semble ne se présenter toujours qu'en creux, comme le contrepied exact des positions des «formalistes » (c'est-à-dire misésiennes). Si le formalisme implique une "pure logique du choix", alors le " substantivisme » devra, au contraire être " empiriste »; quand le formalisme échoue à interroger les fins des individus (préférant se consacrer aux moyens), le 
substantivisme devra concerner le «bien-vivre » de l'individu dans son groupe social : ces antinomies systématiques sont nombreuses. L'opposition, même justifiée sur le plan épistémologique (par un réalisme revendiqué face au réductionnisme formaliste), même légitimée moralement - au regard de valeurs, dont certaines d'inspiration religieuse chrétienne plus encore que socialiste -, ne constitue cependant pas un paradigme comme tel, ni une position en mesure de répondre à la structure cohérente élaborée par Mises dès 1920, puis surtout en 1949 avec Human Action.

Pour des raisons qui n'excluent pas un certain opportunisme et le goût de la polémique, Polanyi a laissé dans la lignée "autrichienne " une œuvre riche d'intuitions et inaboutie à la fois. Le paradigme qu'il cherchait à Vienne lorsqu'il rencontra Mises, Polanyi le trouva avant de s'établir à New York en relisant Weber. Faute peut-être de n'avoir pas saisi plus tôt l'ampleur de cette sociologie, Polanyi voulut compléter de son côté des recherches monographiques déjà formulées un demi-siècle plus tôt, notamment concernant les économies antiques ${ }^{45}$. Cependant, Polanyi n'a pas repris, dans son propre appareil conceptuel, l'entièreté des concepts exposés dans le premier chapitre de Wirtschaft und Gesellschaft, ni les analyses wébériennes de la domination, des communautés de types divers, ni les études sur la ville. Et il a puisé, mais de même incomplètement, dans la seconde édition des Grundsätze der Volkswirtschaftslehre de Menger. Le résultat de l'intégration non systématique de concepts de ces œuvres est une esquisse de paradigme abondant en aperçus intuitifs, mais au final moins structurée que les travaux posthumes, datant du début des années 1920, dont elle s'inspire. Il reste que rapprocher Menger et Weber fournissait un cadre (que nous qualifions de "vétéro-autrichien ») qui replace la tradition autrichienne sur un socle notionnel plus solide et plus large.

\section{Conclusion}

Indiquons pour finir que Polanyi fut le premier sans doute, mais non le seul, à voir la possibilité d'un rapprochement entre l'école autrichienne et Weber. Des années plus tard, Ludwig Lachmann allait publier sa thèse d'économie " autrichienne ", The Legacy of Max Weber (1971), en l'inscrivant toutefois dans le cadre distinct de l'institutionnalisme nord-américain. Inversement, aucun collègue ni élève de Polanyi à l'université Columbia ne reprenait ses réflexions sur les «deux sens" de l'économie. Seul Schafer, à qui fut confiée entre 1967 et 1973, la traduction en allemand de The Great Transformation, resta fidèle à l'esprit de son ami.

Or ce camarade de l'époque viennoise avait déjà réuni, dans son exil en NouvelleZélande, des textes de Mises et Böhm-Bawerk ${ }^{46}$ afin d'établir les correspondances terminologiques entre l'anglais et l'allemand. Dans sa correspondance avec la veuve de Polanyi (et héritière officielle de son œuvre), une dispute s'éleva sur l'influence de Polanyi à Vienne sur la pensée de Popper; ce dernier, dans son Open Society and its Enemies, reconnaissait devoir à Polanyi sa conception du «nominalisme méthodologique $^{47}$ ». Or Popper influença Hayek sans doute autant que Mises, de sorte qu'un nœud de relations lie ces penseurs critiques de la rationalité formelle, qui trouveraient leur place dans un paradigme "vétéro-autrichien ». Polanyi y représente le penseur d'une rationalité "substantive»: il penche pour les raisons éthiques sans doute, mais les enjeux sont épistémologiques. 
doute trop soucieux de son débat entamé à Vienne avec Mises, Polanyi n'a sans doute pas su exploiter au mieux dans sa période nord-américaine, notamment en préparant les textes que Livelihood of Man réunit à titre posthume, les potentialités qu'offraient les œuvres (elles aussi posthumes) de Weber et Menger. Aujourd'hui, près d'un siècle après ces textes, et à un demi-siècle de Polanyi, la tâche de chercheurs dignes de ces recherches laissées inachevées peut consister à les articuler dans un ensemble qui tirerait d'elles sa force. Et où elles seraient plus que jamais vivantes.

\section{BIBLIOGRAPHIE}

N.B. Les références ci-dessous s'ajoutent à celles qui figurent dans les notes.

\section{Sources}

Notes préparatoires de Karl Polanyi et Felix Schafer « Hans Mayer's Lösung des Zurechnungsproblems », Montréal, Université Concordia, Karl Polanyi Institute of Political Economy, Karl Polanyi Digital Archive, 02/10*.

Karl Polanyi, « Carl Menger's Two Meanings of "Economic” », Montréal, Université Concordia, Karl Polanyi Institute of Political Economy, Karl Polanyi Digital Archive, 42/09*.

\section{Bibliographie}

MENGER Carl, Grundsätze der Volkswirtschaftslehre, Wien, Hölder-Pichler-Tempsky, 1923.

MISES Ludwig von, « Die Wirtschaftsrechnung im sozialistischen Gemeinwesen », Archiv für Sozialwissenschaften, vol. 47, 1920.

MISES Ludwig von, Die Gemeinwirtschaft. Untersuchungen über den Sozialismus, Jena, Gustav Fischer, 1932.

MISES Ludwig von, Human Action. A Treatise on Economics, Indianapolis, Liberty Fund, 1949 [Ebook edition, 2011].

POLANYI Karl, « Sozialistische Rechnungslegung », dans Claus Thomasberger et Michele Cangiani, Chronik der großen Transformation, Marburg, Metropolis, 2005, vol. 3, p. 71-113.

POLANYI Karl, « Die funktionelle Theorie der Gesellschaft und das Problem der sozialistischen Rechnungslegung ", dans Claus Thomasberger et Michele Cangiani, Chronik der großen Transformation, Marburg, Metropolis, 2005, vol. 3.

POLANYI Karl, « Neue Erwägungen zu unserer Theorie und Praxis », dans Claus Thomasberger et Michele Cangiani, Chronik der großen Transformation, Marburg, Metropolis, 2005, vol. 3, p. 114-125. POLANYI Karl, « The Essence of Fascism », Economy and Society. Selected writings, Claus Thomasberger et Michele Cangiani (éd.), Cambridge, Polity Press, 2018 [1935], p. 81-109. 
WEBER Max, Wirtschaft und Gesellschaft. I. Soziologie. Unvollendet 1919-1920, Knut Borchardt et Jürgen Deininger (éd.), Tübingen, J. C. B. Mohr, « Studienausgabe der Max-Weber-Gesamtausgabe, 1, 23 », 2014.

\section{NOTES}

1. Voir l'ensemble de fragments autobiographiques rassemblés dans la boîte 30 , dossier 02 (désormais 30/02) des Archives Karl Polanyi, conservées au Karl Polanyi Institute of Political Economy de l'université Concordia à Montréal. Ces archives, pour l'essentiel accessibles en ligne (https://www.concordia.ca/research/polanyi/archive.html), constituent le Karl Polanyi Digital Archive (désormais KPDA). Quand les références d'archives sont suivies d'un astérisque, le texte est consultable en « plein texte » sur http://karl.polanyi.fr/wiki/.

2. Voir KPDA, Erinnerungen de Felix Schafer, 1964-1966, 29/09 et Karl Polanyi's Life in Vienna Memoirs de Felix Schafer, 1973-1974, 29/10.

3. Polanyi écrit avoir trouvé la foi chrétienne sur le front est en 1918, et il s'est converti officiellement en 1919. Cette même année, il travaille pour l'éphémère gouvernement communiste hongrois, après avoir participé au régime libéral et radical du comte Károlyi.

4. Voir Gareth Dale, Karl Polanyi: A Life on the Left, New York, Columbia University Press, 2016, p. 72. Notre traduction des textes anglais, sauf précision contraire.

5. Voir l'ouvrage de Jörg Guido Hülsmann, Mises: The Last Knight of Liberalism, Auburn, Ludwig von Mises Institute, 2007, p. 331.

6. Jörg Guido Hülsmann, op. cit., p. 342-343. La suite du texte ne laisse aucun doute sur le fait que le « $\mathrm{D}^{\mathrm{r}}$. Polanyi » mentionné est bien Karl Polanyi. On est sans renseignements notables sur le « $\mathrm{D}^{\mathrm{r}}$. Görög ». Toutes les traductions de l'anglais sont de notre fait.

7. Schafer est alors étudiant. Il écrit en 1926 sa thèse sur ce thème de l'objectivation: «Über Staats- und Rechtsbegriffe und ihre Verselbständigung " où il reconnaît tout ce qu'il doit à Polanyi.

8. Lettre du 24 février 1924 de Karl Polanyi à Ludwig von Mises, Auburn, Mises Institut, Ludwig von Mises Archives, 80/8*.

9. Polanyi semble plus ouvert à l'austro-marxisme d'un Max Adler, par exemple.

10. Voir en particulier Karl Polanyi, "The Essence of Fascism ", Economy and Society. Selected writings, Claus Thomasberger et Michele Cangiani (éd.), Cambridge, Polity Press, 2018 [1935], p. 105.

11. Polanyi se souvenait très bien de Mises. Il écrivait ainsi au mentor de sa période hongroise, Oszkár Jászi, dans une lettre du 1er mars 1943: "Je ne crois pas dans une économie totalement planifiée. Mon premier article sur l'économie socialiste, il a quelque vingt ans, partait de là. Mises m'avait attaqué, en disant que je nourrissais des illusions, si je pensais qu'il existait une voie entre le laissez-faire et une économie totalement planifiée. Aujourd'hui, nous savons bien ce qui est arrivé à Mises et à son point de vue. En réalité, on ne voit aujourd'hui rien d'autre que des modèles à cheval entre les deux » (cité dans György Litván, « Democratic and Socialist Values in Karl Polanyi's Thought », dans Marguerite Mendell et Danielle Salée [dir.], The Legacy of Karl Polanyi, London, MacMillan, 1991, p. 259). Les italiques sont de Polanyi.

12. Karl Polanyi, The Great Transformation. The Political Economic Origins of our Time, Boston, Beacon Press, 2001, chap. 12, http://karl.polanyi.fr/wiki/index.php? title=The_Great_Transformation\#p148, p. 148, notre traduction.

13. Voir KPDA, plan du l'ouvrage de Karl Polanyi sur les « Origins of the Cataclysm », 1943; 19/05 (daté de 1941). 
14. Plusieurs documents de 1943 l'attestent, dont le contrat avec son éditeur. Voir KPDA, Contrat entre Karl Polanyi, Farrar et Rinehart pour la publication de The Great Transformation, 1943-1944, $13 / 04$.

15. Y compris les réalités menaçantes comme la bombe atomique en cours de réalisation en 1944, ouvrant une nouvelle ère qui inquiète alors chaque penseur politique majeur.

16. Scansions historiques discutables qui doivent sans doute remonter au $\mathrm{XVI}^{\mathrm{e}}$ siècle et à l'humanisme tant prisé par Polanyi bien qu'il ait fissuré l'unité organique de la civilisation européenne, ce bien avant l'industrialisation.

17. Voir la lettre à Michael Polanyi du 6 janvier 1947, citée par Gareth Dale, op. cit., p. 232. Polanyi connaissait son œuvre dès son passage à Vienne. Un carnet de notes, probablement de 1921, atteste qu'il y a étudié les Gesammelte Aufsätze zur Religionssoziologie. Voir KPDA, Notes de lecture de Karl Polanyi durant sa période viennoise aux débuts des années 1920, 04/10, p. 100-131).

18. Polanyi ajoute Wirtschaft und Gesellschaft dans une liste de textes sur les sociétés non industrielles qu'il devait lire (liste tapée à la machine à écrire, présente dans les archives). Cela semble indiquer que Polanyi n'avait d'abord pas pris en compte cette œuvre pourtant majeure. Au début de 1947, Polanyi écrit à son épouse, depuis New York, qu'il cherche à se procurer ce livre, devenu rare et cher (KPDA, Correspondance entre Karl Polanyi et Ilona Duczynska, 1943-1947, 59/08, p. 481). On sait enfin par ailleurs que Polanyi possédait aussi les Gesammelte Aufsätze zur Wissenschaftslehre déjà à Vienne (KPDA, Gesammelte Aufsätze zur Wissenschaftslehre de Max Weber, 1922, 03/23).

19. http://karl.polanyi.fr/wiki/index.php?title=The_Great_Transformation\#p48, p. 48.

20. Le 5 janvier 1958, Karl Polanyi écrit à son frère Michael avoir écrit une lettre de 17 pages (qui a dû se perdre) où il développait quelques idées issues de son " ancienne conversation " avec Mises. Il évoque « le casse-tête [conundrum] de Mises » pour parler du défi des années 1920 lancé par l'Autrichien sur le calcul en économie socialiste. Voir KPDA, Correspondance entre Karl Polanyi et Michael Polanyi, 1943-1961, 57/08, p. 31-32.

21. Il s'agissait des ouvrages de Mises : Theorie des Geldes und der Umlaufsmittel (1912), Liberalismus (1927) et Nationalökonomie (1940). Polanyi a sans doute également pris connaissance de Die Gemeinwirtschaft (1922).

22. Weber citait élogieusement Mises et Böhm-Bawerk, dans Wirtschaft und Gesellschaft (chap. 1, $\S 9$ ). Il avait également demandé une contribution à Wieser pour ce volume qui la donna, mais qui fut finalement écartée. Polanyi range Weber du côté de ceux qu'il va appeler les "formalistes ». Pourtant, l'adhésion de Weber au "libéralisme» et à la notion de rationalité formelle apparaissait surtout par défaut: le sociologue allemand semblait anticiper dans une certaine mesure un pessimisme culturel - que Polanyi déploie lui aussi en 1944 dans The Great Transformation - lorsqu'il parle des sectes protestantes au $\mathrm{XVI}^{\mathrm{e}}$ siècle à l'origine du "stahlhartes Gehäuse » dans le comportement économique à partir du XviII ${ }^{\mathrm{e}}$ siècle (traduit inexactement par Talcott Parsons dans la version anglaise par iron cage).

23. Le modèle le plus proche de ces exigences qu'ait trouvé Polanyi était les communautés industrielles créées par l'Anglais Robert Owen. Malheureusement, Polanyi n'a achevé ni Freedom and Technology ni une nouvelle version de The Great Transformation, entre 1954 et 1961, où des chapitres entiers devaient être consacrés à ses réalisations.

24. Max Weber, Wirtschaft und Gesellschaft, Werner Gephart et Siegfried Hermes (éd.), Tübingen, J.C. B. Mohr, 2014, vol. 1, 350 p. Voir les notions de «Formale Rationalisierung und Materiale Rationalisierung des Rechts ", p. 115-131.

25. Ce point délicat semble d'ailleurs contredire la critique de la séparation entre l'économie et le politique, que Polanyi formule dès les années 1930, alors que l'économie est, pour lui, un sousensemble d'activités indissociable des autres types d'activités sociales. 
26. Max Weber, Wirtschaft und Gesellschaft. I. Soziologie. Unvollendet 1919-1920, Knut Borchardt et Jürgen Deininger (éd.), Tübingen, J. C. B. Mohr, « Studienausgabe der Max-Weber-Gesamtausgabe, 1,23 », 2014, chap. 2, §9, p. 60.

27. Notons que les termes s'entremêlent entre des œuvres toutes demeurées inachevées à différents égards: Wirtschaft und Gesellschaft de Weber, mais aussi la seconde édition des Grundsätze der Volkswirtschaftslehre éditée par le fils de Menger en 1923 (sans une partie des notes paternelles, voir Gilles Campagnolo, «Menger: from the Works published in Vienna to his Nachlass ", dans Gilles Campagnolo [dir.], Carl Menger. Discussed on the Basis of New Findings, Frankfurt/Main-Wien, Peter Lang, 2008, p. 31-58), et enfin le recueil de textes de Polanyi réunis sous le titre The Livelihood of Man, Harry Pearson (éd.), New York, Academic Press, 1977.

28. Ce faisant, il suit, d'une part, l'usage de son ami Karl Mannheim qui, en 1935, dans Mensch und Gesellschaft im Zeitalter des Umbaus distinguait deux significations (zwei Bedeutungen) de rationalité et d'irrationalité, qu'il nommait «fonctionnelle » et « substantielle »- opposition reprise dans la version augmentée en anglais Man and Society in an Age of Reconstruction (1940) (functional/ substantial). Là aussi l'inspiration wébérienne est claire. Un carnet de notes de Polanyi de 1934-1935 (KPDA, Notes de lecture de Karl Polanyi, 1934-1946, 07/08, p. 1-52, où Weber est cité) montre que Polanyi a étudié le texte allemand de Mannheim. D'autre part, les sociologues américains Talcott Parsons et A. R. Henderson, en publiant en anglais la première partie de Wirtschaft und Gesellschaft (1947, intitulé The Theory of Social and Economic Organization), avaient eux aussi traduit cette distinction par « formal vs substantive ». Les archives de Polanyi montrent qu'il use comme synonymes de «substantif », «sociétal », « institutionnel » (KPDA, Karl Polanyi: Interdisciplinary Project: "Economic Aspects of Institutional Growth" - Ford Foundation Columbia University, New York - Applications for grants and budget statements, 1948-1962, 31/15, p. 64] voire «empirique » (« économie empirique »). Voir KPDA, Première version de Livelihood of Man - table des matières, préface et introduction, 1950-1955, 35/11, p. 84.

29. On peut également trouver une note " $c f$. Menger ", en marge d'un passage écrit à la main et difficile à déchiffrer, mais qui se termine par « la première position fut de manière conséquente défendue par Menger, Robbins, etc., et la seconde par moi ». Voir KPDA, Notes de lecture de Karl Polanyi, Notes de Karl Polanyi - «Origins of Institutions », 1934-1946, 07/09, p. 112.

30. Ceci a l'avantage d'éviter toute confusion avec le matérialisme, notamment celui de philosophies monistes de la science (comme celle d'Ernst Mach), mais aussi, bien entendu, le matérialisme historique marxiste.

31. On voit dans les différents brouillons de textes des années 1950 que Polanyi a besoin de voir dans la distinction mengerienne un apport révolutionnaire pour pouvoir se dire l'unique héritier d'un si grand trésor oublié. Pourtant, cette distinction, introduite lors des travaux réalisés en vue d'une deuxième édition de ses Grundsätze, faisait, sous un entrelacs d'appellations diverses, déjà partie du Zeitgeist de l'époque de Menger.

32. En anglais, c'est meaning que choisit Polanyi pour ce que Menger désignait par Disposition ou Richtung : la terminologie restait peu fixée, à l'état de brouillon dans les archives du Nachlaß de Menger. En fait Bedeutung serait exact, mais Menger l'emploie par ailleurs dans le raisonnement marginaliste, et pas pour ce que vise Polanyi (précision due à Gilles Campagnolo). Les «types » wébériens et ces «sens" repris de Menger sont caractérisés identiquement : ils participent du même Zeitgeist de la sociologie économique de leur époque.

33. En effet, sous l'effet des choix opérés par Friedrich Hayek en 1934 et Lionel Robbins en 1951, la seconde et problématique édition des Grundsätze der Volkswirtschaftslehre de 1923, éditée par le fils de Menger sur la base d'une partie limitée des brouillons de son père, n'avait pas été traduite en anglais (Gilles Campagnolo, op. cit.) En outre, elle était difficile, sinon impossible, à trouver dans les années 1950 aux États-Unis. L'édition de 1871 avait été réimprimée, mais en allemand, par les soins de Hayek ; la première traduction anglaise parut en 1951. 
34. Polanyi avait élaboré cette «triade » dans une conférence de 1930 intitulée « Einführung in die Volkswirtschaftslehre» (KPDA, Tapuscrit de la conférence «Einführung in die Volkswirtschaftslehre » de Karl Polanyi, 1930-1931, 02/20), sans encore faire appel à la notion de catallactique. Reprise, l'expression devenait au fond synonyme du reproche de "formalisme». Ajoutons que Polanyi prétend reprendre le terme "catallactique» (comme Mises le dit de son côté) à un obscur évêque du $\mathrm{xIx}^{\mathrm{e}}$ siècle, Richard Whately. Cela paraît douteux. Polanyi reste discret sur la provenance du mot, qu'il utilise à foison entre 1947 et 1960 sans l'expliquer, comme l'atteste l'interrogation portée par George Dalton, l'un de ses plus proches collaborateurs à New York. Dans une lettre de 1961, soit quatorze ans après que Polanyi a commencé à utiliser le terme, et quelques mois après s'être lui-même fait admonester pour sa «défense de la redistribution comme une sorte d'échange» (ce qui, pour Polanyi «n'est pas seulement [une façon de voir] catallactique, mais dans sa variante benthamienne, utilitariste " [KPDA, Correspondance de Karl Polanyi, mai-août 1961, 52/01, p.60]), Dalton demande « une bonne définition de catallactique» (ibid., p. 121), preuve s'il en est que Polanyi restait évasif sur ce point.

35. Sur la catallactique, ou «science des échanges ", voir l'ensemble de la quatrième partie de Human Action.

36. Indiquons à cet égard que les auteurs autrichiens soulignent, comme Hayek, la nature violente de ce fonctionnement communautaire dans les groupes « tribaux ».

37. Version reprise en anglais et largement augmentée de son livre Nationalökonomie publié en 1940.

38. Le volume collectif Trade and Markets in the Early Empires, que Polanyi codirige, constitue une sorte de manifeste du courant substantiviste en anthropologie. Pour saisir son histoire éditoriale complexe, voir Karl Polanyi, Conrad M. Arensberg et Harry W. Pearson, Commerce et marché dans les premiers empires. Sur la diversité des économies, Michele Cangiani et Jérôme Maucourant (éd.), Lormont, Le Bord de l'eau, 2017, et par exemple sa recension par Patrick Gilormini dans la Revue de philosophie économique, vol. 20, juin 2019, p. 157-167.

39. Voir dans ce volume, la présentation de Christel Vivel.

40. Voir Gilles Campagnolo, "Seuls les extrémistes sont cohérents...": Rothbard et l'École austroaméricaine dans la querelle de l'herméneutique, Lyon, ENS, 2006.

41. Une autre possibilité serait "paléo-autrichien », l'image cependant serait celle d'une "préhistoire » alors qu'il s'agit de retourner aux premières armes de cette école.

42. La version éditée par George Dalton en 1977 (donc également posthume) présente, selon nous, le meilleur état de la réflexion polanyienne des années 1947-1953.

43. Karl Polanyi, The Livelihood of Man, Harry Pearson (éd.), New York, Academic Press, 1977, p. 19. Version anglaise : http://karl.polanyi.fr/wiki/index.php?title=The_Livelihood_of_Man\#p19.

44. Karl Polanyi, "The Economy as Instituted Process ", dans Karl Polanyi, Conrad Arensberg et Harry Pearson (dir.), Trade and Market in the Early Empires. Economies in History and Theory, Glencoe, The Free Press-The Falcon's Wing Press, 1957, p. 243. Version anglaise : http://karl.polanyi.fr/ wiki/index.php?title=The_Economy_as_Instituted_Process\#p243.

45. Voir notamment de Weber: Zur Geschichte der Handelsgesellschaften im Mittelalter (1889), Die römische Agrargeschichte in ihrer Bedeutung für das Staats- und Privatrecht (1891), Die sozialen Gründe des Untergangs der antiken Kultur (1896).

46. Voir Université Concordia (Montréal), Karl Polanyi Digital Archive, Notes explicatives de Felix Schafer à propos de la traduction de ses propres mémoires en anglais, n. d., 29/11.

47. Karl Popper, Open Society and its Enemies [1945], Londres, Routledge Classic, 2005, vol. 1, p. 232. Sur la polémique indiquée, voir une lettre de Schafer à Ilona Duczyńska d'août 1973 (KPDA, Correspondance entre Adele et Felix Schafer et Ilona Duczynska, 1964-1978, 58/11, p. 93-94,) suscite une réplique de cette dernière où elle nie toute influence de son mari sur « le prophète du libéralisme » (ibid., p. 96-97). On ne sait rien d'une éventuelle rencontre entre Schafer et Popper 
en Nouvelle-Zélande entre juin 1939 (KPDA, Karl Polanyi's Life in Vienna - Memoirs de Felix Schafer, $1973-1974,29 / 10$, p. 74) et 1945, lorsqu'ils s'y trouvaient tous deux en exil. En outre, dans ses deuxièmes mémoires, Schafer dit que c'est de Polanyi que Popper tenait l'idée d'une unité de méthode entre sciences sociales et sciences naturelles (ibid., p. 52).

\section{RÉSUMÉS}

Les analyses de Felix Schafer, alors le plus proche collaborateur de Karl Polanyi (1886-1964), permettent de comprendre un débat entamé en 1922 à Vienne entre Polanyi et Ludwig von Mises (qui était alors le représentant le plus célèbre de l'école autrichienne). Les enjeux de leur opposition devaient structurer la pensée polanyienne en son entier. Dans les décennies suivantes, notamment les années 1940-1950 aux États-Unis, c'est en esquissant une approche de type sociologique que Polanyi allait paradoxalement élaborer, sur des catégories conjointes qu'il tire indissociablement de Max Weber et de Carl Menger, un paradigme que nous qualifions de « vieux (ou vétéro)-autrichien » en raison de son retour aux sources de cette tradition (prise au sens large). Le formalisme de Mises s'y intègre pour former une «branche formaliste » selon Polanyi, qui nous a peut-être laissé là sa plus féconde intuition.

Die von Felix Schafer, seinem damals engsten Mitarbeiter, ausgearbeitet Analyse zeigt, wie die 1922 in Wien begonnene Debatte mit Ludwig von Mises in den folgenden Jahrzehnten das ganze Denken von Karl Polanyi (1886-1964) strukturiert hat. In den 1940er und 1950er Jahren skizzierte er in den Vereinigten Staaten von Amerika eine soziologische Herangehensweise, die paradoxerweise auf den miteinander verbundenen und untrennbaren Kategorien der kontinentalen Europäer Max Weber und Carl Menger basiert. Mit diesem Paradigma, das wir auf Grund seiner Rückkehr zu den Quellen der österreichischen Tradition in einer erweiterten Version als ein „alt-österreichisches“ qualifizieren können und in dem von Mises' Formalismus integriert ist, hat Polanyi uns das Ergebnis seiner fruchtbarsten Intuition hinterlassen.

Thanks to Karl Polanyi's closest collaborator during his Austrian Period, Felix Schafer, we can understand how the debate with Ludwig von Mises - begun in 1922 in Vienna - structures the following decades of Polanyi's thinking, and particularly in the 1940s and 1950s. Indeed, paradoxically, as Polanyi is in the USA, it is by sketching a sociological paradigm based on the conjoined and inseparable categories of continental Europeans Max Weber and Carl Menger paradigm that we call for his return to the sources of the Austrian tradition in an extended version, "Old(veterus)-Austrian" -, in which the formalism of von Mises is integrated as the formalist branch, Polanyi has left us his most fruitful intuition.

\section{INDEX}

Mots-clés : Mises (Ludwig von), Polanyi (Karl), Weber (Max), substantivisme

Schlüsselwörter : Mises (Ludwig von), Polanyi (Karl), Weber (Max), substantivismus

Keywords : Mises (Ludwig von), Polanyi (Karl), Weber (Max), substantivism 
AUTEUR

SANTIAGO PINAULT

Brunel University, London 